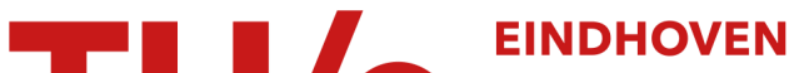 \\ UNIVERSITY OF \\ TECHNOLOGY
}

\section{Luminescent metamaterials for solid state lighting}

Citation for published version (APA):

Nikitin, A., Remezani, M., \& Gómez Rivas, J. (2016). Luminescent metamaterials for solid state lighting. ECS Journal of Solid State Science and Technology, 5(1), R3164-R3169. https://doi.org/10.1149/2.0211601jss

DOI:

10.1149/2.0211601jss

Document status and date:

Published: 01/01/2016

\section{Document Version:}

Publisher's PDF, also known as Version of Record (includes final page, issue and volume numbers)

\section{Please check the document version of this publication:}

- A submitted manuscript is the version of the article upon submission and before peer-review. There can be important differences between the submitted version and the official published version of record. People interested in the research are advised to contact the author for the final version of the publication, or visit the $\mathrm{DOI}$ to the publisher's website.

- The final author version and the galley proof are versions of the publication after peer review.

- The final published version features the final layout of the paper including the volume, issue and page numbers.

Link to publication

\section{General rights}

Copyright and moral rights for the publications made accessible in the public portal are retained by the authors and/or other copyright owners and it is a condition of accessing publications that users recognise and abide by the legal requirements associated with these rights.

- Users may download and print one copy of any publication from the public portal for the purpose of private study or research.

- You may not further distribute the material or use it for any profit-making activity or commercial gain

- You may freely distribute the URL identifying the publication in the public portal.

If the publication is distributed under the terms of Article 25fa of the Dutch Copyright Act, indicated by the "Taverne" license above, please follow below link for the End User Agreement:

www.tue.nl/taverne

Take down policy

If you believe that this document breaches copyright please contact us at:

openaccess@tue.nl

providing details and we will investigate your claim. 
JSS Focus Issue on Novel Applications of Luminescent Optical Materials

\title{
Luminescent Metamaterials for Solid State Lighting
}

\author{
Andrey Nikitin, Mohammad Remezani, and Jaime Gómez Rivas ${ }^{*, z}$ \\ FOM Institute for Atomic and Molecular Physics, c/o Philips Research, 5656 AE Eindhoven, The Netherlands
}

\begin{abstract}
We investigate the luminescent spectrum of thin films of dye molecules embedded in a polymer layer and on top of an array of aluminum nanoparticles. The emission couples to quasi-guided modes that are efficiently coupled out to free space by scattering with the particle array. This outcoupling provides a significant enhancement of the emission in defined directions. The mode density changes and the spectrum is modified by varying the thickness of the layer. In consequence, the luminous efficacy of the layer of dye can be controlled by the layer thickness and the characteristics of the particle array. This system can be regarded as a luminescent metamaterial with designed properties that can be exploited in solid state lighting applications.

(C) The Author(s) 2015. Published by ECS. This is an open access article distributed under the terms of the Creative Commons Attribution 4.0 License (CC BY, http://creativecommons.org/licenses/by/4.0/), which permits unrestricted reuse of the work in any medium, provided the original work is properly cited. [DOI: 10.1149/2.0211601jss] All rights reserved.
\end{abstract}

Manuscript submitted July 20, 2015; revised manuscript received September 23, 2015. Published October 16, 2015. This paper is part of the JSS Focus Issue on Novel Applications of Luminescent Optical Materials.

The invention of the efficient blue Light Emitting Diode (LED) ${ }^{1}$ has led to the Solid State Lighting (SSL) revolution that will replace inefficient incandescent and fluorescent lamps by much more efficient light sources. Blue light is necessary for the generation of broadband white light. In a white LED this generation is usually realized through a luminescent or phosphorescent material, which is called the phosphor. ${ }^{2}$ Blue light from efficient InGaN quantum wells is absorbed by the phosphor, which emits light of a longer wavelength. White light is generated by mixing the emission of the phosphor with the fraction of non-absorbed blue light. Research on light generation in white LEDs has mainly focused on the improvement of the quantum efficiency of the phosphor by modifying the material composition and on the change of the emission spectrum in order to optimally match it to the photopic sensitivity curve of the eye. ${ }^{3}$

In this article we demonstrate a radically different approach to modify the emission intensity and spectrum of phosphors for SSL. This approach relies on coupling the emission to quasi-guided modes in the phosphor layers. These modes are very efficiently outcoupled to free space radiation by scattering with periodic arrays of metallic nanoparticles. Metallic nanoparticles support localized surface plasmon polaritons (LSPPs), which are the coherent oscillations of the free charges in the metal driven by the electromagnetic field. ${ }^{4}$ LSPPs are resonant phenomena leading to the increase of the polarizability of the particle and, consequently, to the scattering efficiency. ${ }^{5}$ In order to use resonant plasmonic nanoparticles in real applications, we need to have large arrays or ensembles of these nanostructures. Therefore, we investigate the modification of the emission from phosphor layers on top of periodic arrays of metallic nanoparticles. These and similar arrays have received recently a significant attention because of their capacity of supporting collective plasmonic resonances. These collective resonances are the result of the enhanced radiative coupling of LSPPs through scattering and diffraction by the particle array. Two different kinds of collective resonances in periodic arrays of plasmonic particles have been described in the literature. ${ }^{6}$ The first kind arises in waveguides with the particle array in their proximity (usually on one facet). The guided modes are responsible of the enhanced radiative coupling of LSPPs. ${ }^{7,8}$ Alternatively, this system can be regarded as a waveguide supporting quasi-guided modes that are coupled to freespace radiation by scattering with the particle array. The second kind of collective resonances arises from the in-plane diffracted orders of the array, ${ }^{9-11}$ and it does not require a waveguiding layer.

Light emission from emitters in the proximity of these systems has been recently reported. ${ }^{12-19}$ Collective plasmonic resonances lead to the enhanced and directional emission that has been proposed for the

\footnotetext{
*Electrochemical Society Active Member.
}

${ }^{\mathrm{z} E}$-mail: j.gomezrivas@ differ.nl improvement of the performance of white LEDs. ${ }^{20-22}$ Here, we report on the forward emission from quasi-guided modes in polymer layers containing dye molecules. We describe how the emission spectrum of the system is changed by varying the thickness of the waveguide. This change in thickness modifies the number of guided modes that can be supported by the structure. As the emission spectrum of the dye depends on the mode distribution, this emission is controlled and can be increased by the layer thickness. In this way, the luminous efficacy of the system is not only defined by the phosphor internal structure, but also by geometrical parameters, such as the waveguide thickness and the particle array. These parameters can be easily changed to achieve a certain emission. Due to these characteristics, we refer to these systems as a luminescent metamaterials, i.e., engineered composite materials that exhibit superior properties not observed in the constituent materials or nature.

\section{Sample Fabrication and Spatial Distribution of the Emission}

We have fabricated a periodic array of aluminum (Al) nanoparticles using substrate conformal nanoimprint lithography (SCIL). ${ }^{23} \mathrm{~A}$ rubber stamp is used with this technique to transfer a pattern onto a substrate (silica in our case). This pattern is used in combination with metal evaporation and lift-off to define large arrays of nanoparticles. The advantage of SCIL over other nanofabrication techniques, such as electron beam lithography or focus ion beam lithography, is that it enables the fast structuring of large surfaces with an excellent reproducibility. A scanning electron microscope image of the fabricated array of nanoparticles is shown in Fig. 1a. The array is a hexagonal lattice with a pitch of $450 \mathrm{~nm}$. Each nanoparticle has a pyramid shape with a height of $150 \mathrm{~nm}$ and a top and bottom diameters of 70 and 140 $\mathrm{nm}$, respectively. We have chosen $\mathrm{Al}$ as the metal for the fabrication of the nanoparticles because it is an unexpensive material with relatively low losses in the visible spectrum. ${ }^{24}$ These characteristics make $\mathrm{Al}$ an excellent candidate for solid-state lighting applications.

As a luminescent layer we have used two different organic molecules, namely, Lumogen F Red 305 (F305) and Lumogen F Orange 083 (F083) from BASF, embedded in polystyrene (PS). These dyes are characterized by their photostability and are suitable phosphors for white light generation in solid state lighting. The concentrations of F305 and F083 are $4 \mathrm{wt} \%$ and $2 \mathrm{wt} \%$ of the polymer matrix, respectively. This mixture of organic dyes exhibits an efficient Förster resonant energy transfer (FRET). Figure 1b displays the absorption and emission spectra of the two separate dyes, while Fig. 1c represents these spectra for the mixture. In the FRET process the F083 molecules act as donor and the F305 molecules as acceptor. In this way the absorption at a frequency of $666 \mathrm{THz}(\lambda=450 \mathrm{~nm})$ can be maximized and the red emission optimized. 
(a)
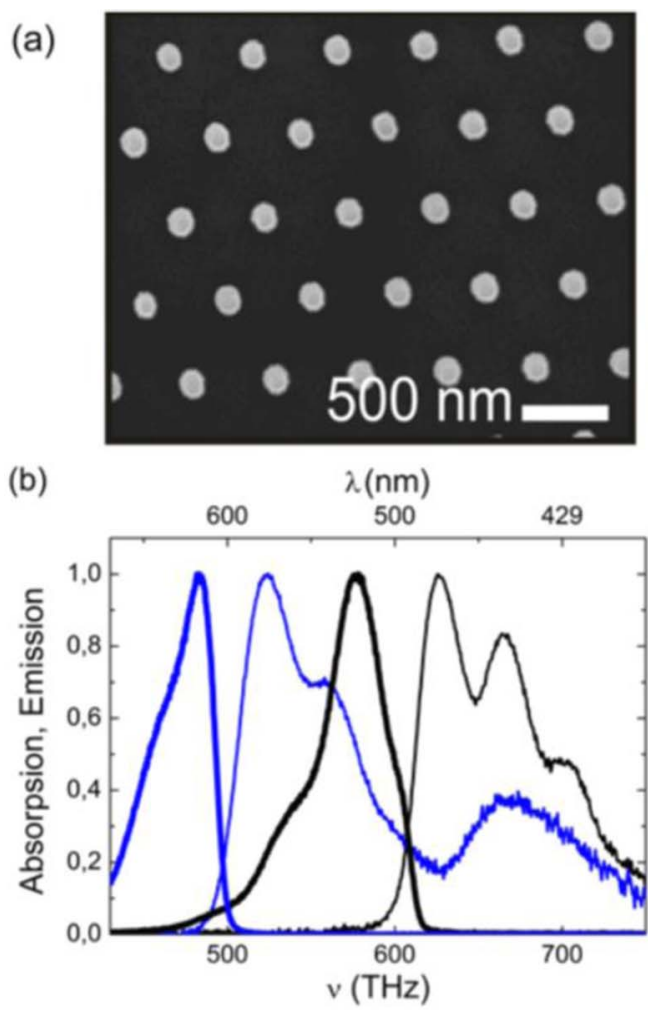

(c)

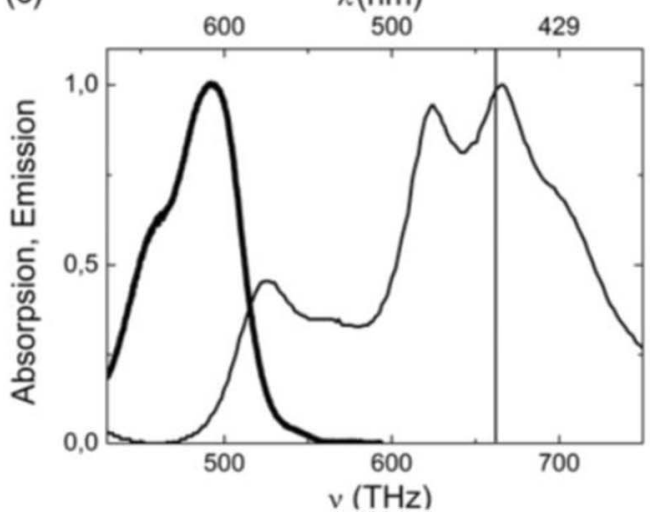

Figure 1. (a) Top view of a scanning electron micrograph of a hexagonal array of $\mathrm{Al}$ nanoparticles with a pitch of $450 \mathrm{~nm}$. (b) Normalized absorption (thin curves) and emission (thick curves) spectra of Lumogen F Red 305 (F305, blue curves) and Lumogen F Orange 083 (F083, black curves) dye embedded in polystyrene. (c) Normalized absorption (thin curve) and emission (thick curve) of the phosphor layer containing the mixture of Lumogen F Red 305 and Lumogen F Orange 083. The vertical line represents the frequency of the excitation laser.

The luminescence layer was deposited on top of the particle array. This was done by dissolving the PS and the organic molecules in cyclopentanone and by spin-coating this solution on top of the array. By varying the viscosity of the solution and the spin rate we could control the thickness of the polymer layer in the range from $t=460$ to $1960 \mathrm{~nm}$ with an uncertainty of $\pm 20 \mathrm{~nm}$. The composite structure, consisting of the array of metallic nanoparticles and the luminescent layer, constitutes the luminescent metamaterial that, as we show below, modifies the emission characteristics (spectrum and direction) of the dye mixture.

As mentioned in the introduction, one of the most relevant characteristics of LSPPs and collective plasmonic resonances is their capacity of enhancing and spatially redistributing the emission of nearby emitters. In order to illustrate this effect, we compare in Fig. 2 the emission from a dye layer with a thickness $t=1550 \mathrm{~nm}$ with the emission of a luminescent metamaterial, i.e., a similar layer on top of the hexagonal array of $\mathrm{Al}$ nanoparticles. The experiment is illustrated in Fig. 2a: We have used a diode pumped continuous wave laser diode emitting at a wavelength of $453 \mathrm{~nm}$. The laser beam is split in two beams with a $50 / 50$ beam splitter to excite with the same optical power of $25 \mathrm{~mW}$ both the reference layer and the luminescent metamaterial. The photoluminescence was collected with bi-convex lenses (focal length $=35 \mathrm{~mm}$ and $\mathrm{NA}=0.34$ ) and projected onto a screen placed at a distance of $70 \mathrm{~mm}$ from the samples. The fraction of the blue light from the laser that was not absorbed was filtered out with two identical band pass filters placed behind the samples with a cutoff frequency of $545 \mathrm{THz}(\lambda=550 \mathrm{~nm})$.

Figure $2 b$ shows a photograph of the emission from the two samples, which are visible in the lower part of the image, projected onto the screen. The reference layer on the left side shows a nearly homogeneous emission over the range of angles collected by the lens. This emission corresponds to a Lambertian emitter. On the other side, the emission from the luminescent metamaterial (right side) is very pronouncedly enhanced and this enhancement is the strongest in the forward direction. The origin of this response has been described in Ref. 20 and it is mainly two-fold: First, the presence of the particle array resonantly enhances the absorption of the blue light in the luminescent layer. This produces an overall enhancement of the emission intensity in all directions. Second, the emission couples to guided modes in the luminescent layer and this emission is coupled out by the array in defined directions. These directions are given by the lattice structure and lattice constant. This is clearly observed in the photograph of the emission, where also a six-fold rotation symmetry in the emission, caused by the hexagonal lattice, is visible. In the example of Fig. $2 b$ these parameters are chosen such that the emission is preferential in a reduced solid angle in the forward direction. Different lattices provide different emission characteristics, such as directional enhancement and luminous efficacy. We also note that periodic lattices of nanoparticles are needed to couple the emission of extended and relatively thick luminescent layers in defined directions. Therefore, random arrays of nanoparticles with same filling fraction as periodic arrays, do not provide similar characteristics in terms of enhanced directional emission. ${ }^{20}$

\section{Layer Thickness Dependence of the Emission}

Emission enhancement. - In order to determine the optimum thickness for the luminescent layer in terms of emission enhancement, we have measured the photoluminescence (PL) of several samples formed by the same $\mathrm{Al}$ particle array but with different dye layer thicknesses on top. Two of these measurements are displayed in Fig. 3. For the measurement the dye was excited with the light from the blue diode laser at normal incidence and the polarization along horizontal direction of Fig. 1a. Figure 3a displays the emission from a luminescent metamaterial with a layer thickness of $t=530 \mathrm{~nm}$, while Fig. $3 \mathrm{~b}$ corresponds to the emission with a layer thickness of $t=1960 \mathrm{~nm}$. In both figures it is also displayed with a red curve the emission spectra of luminescent layer with equal thickness but without the particle array. All the spectra were measured with a fiber coupled spectrometer at the central position of the emission pattern and at the same distance from the sample as the screen used to take the photograph of Fig. 2b. In both panels of Fig. 3 we observe narrow bands of enhanced emission. The width of these bands decreases and their number increases as the luminescent layer thickness increases.

The homogeneous luminescent layer has a refractive index of $n=1.59$ in the visible. This value has been determined from ellipsometry measurements (not shown here). This index is higher than the refractive indexes of the media above and beneath the luminescent layer, which are $n_{1}=1$ and $n_{2}=1.46$. Therefore, the layer supports transverse electric (TE) and transverse magnetic (TM) waveguide modes to which the emission can couple. The dispersion relations of 
(a)

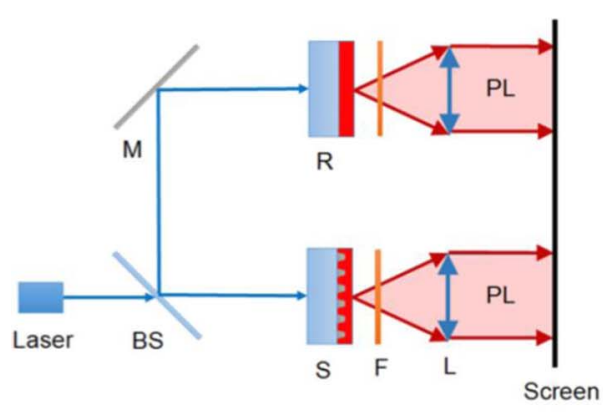

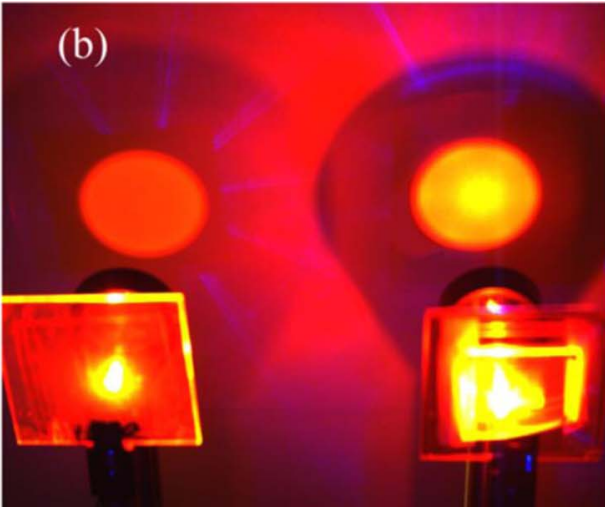

Figure 2. (a) Schematic representation of the optical setup used to generate the image in (b): light from a blue laser diode is split using a 50/50 beam splitter (BS) and directed to the sample (S) consisting of an Al nanoparticle array on top of a silica substrate covered by a dye doped polymer layer, and to the reference (R) sample consisting of a dye layer with the same thickness on top of the silica substrate. The fraction of the laser light that is not absorbed by the sample and reference is filtered using two identical long pass filters $(\mathrm{F})$. The emission from the sample and reference are collimated by two identical lenses (L) and projected onto the screen. (b) Photograph of the emission from the sample (right side) and from the reference (left side).

these modes are given by the following transcendental equations ${ }^{25,26}$

$$
k_{\perp} t=m \pi+\arctan \left(\alpha_{1} / k_{\perp}\right)+\arctan \left(\alpha_{2} / k_{\perp}\right) \quad \text { for TE, and }
$$

$$
k_{\perp} t=m \pi+\arctan \left(\alpha_{1} n^{2} / k_{\perp} n_{1}^{2}\right)+\arctan \left(\alpha_{2} n^{2} / k_{\perp} n_{2}^{2}\right) \text { for TM, }
$$

where

$$
\begin{gathered}
k_{\perp}=\sqrt{\omega^{2} n^{2} / c^{2}-k_{\|}^{2}}, \\
\alpha_{1,2}=\sqrt{k_{\|}^{2}-\omega^{2} n_{1,2}^{2} / c^{2}},
\end{gathered}
$$

$m$ is the order of the mode, $k_{\perp}, \alpha_{1}$ and $\alpha_{2}$ are the transverse wavenumbers in the waveguide, upper medium and substrate respectively, $k_{\|}$is the parallel wavenumber along the propagation direction, and $t$ is the layer thickness.

The number of modes that can be guided in the layer depends on the thickness of the slab; increasing this number as the thickness increases. The particle array beneath the layer provides by scattering the required wavenumber or momentum to the guided light in order to couple out into free space. The large polarizability of the metallic nanoparticles due to the LSPPs enables an efficient scattering and outcoupling of this guided radiation. The vertical lines in Fig. 3 represent the eigenfrequencies of the quasi-guided modes in the periodic structure coupled out in the directional normal to the surface of the sample at which the PL was measured. These eigenfrequencies are calculated using the empty lattice approximation. In this approximation, the dispersion curves of the guided modes are folded into the light cone by scattering with the periodic structure. ${ }^{27}$ This folding back into the light cone results in the coupling of guided modes to free space radiation, which leads to the term quasi-guided when referring to these modes. Note that due to the hexagonal symmetry of the lattice, the modes coupled out in the normal direction have a six-fold degeneracy. As a result the total intensity of the light emitted in this direction can be relatively large.

The two peaks at $504 \mathrm{THz}$ and $495 \mathrm{THz}$ in the emission spectrum of the luminescent metamaterial with the layer thickness $t=530 \mathrm{~nm}$ (Fig. 3a) correspond to the fundamental $\mathrm{TM}_{0}$ and $\mathrm{TE}_{0}$ quasi-guided modes. The measured mode frequencies differ from the calculated eigenfrequencies of the waveguide. These discrepancies can be ascribed to the fact that the eigenfrequencies are calculated using the empty lattice approximation, which assumes no perturbation of the modes by the individual particles forming the lattice and no coupling between modes. This is clearly not the case in view of the relative large size of the particles compared to the thickness of the waveguide. For the layer with a thickness of $t=1920 \mathrm{~nm}$ (Fig. 3b), the
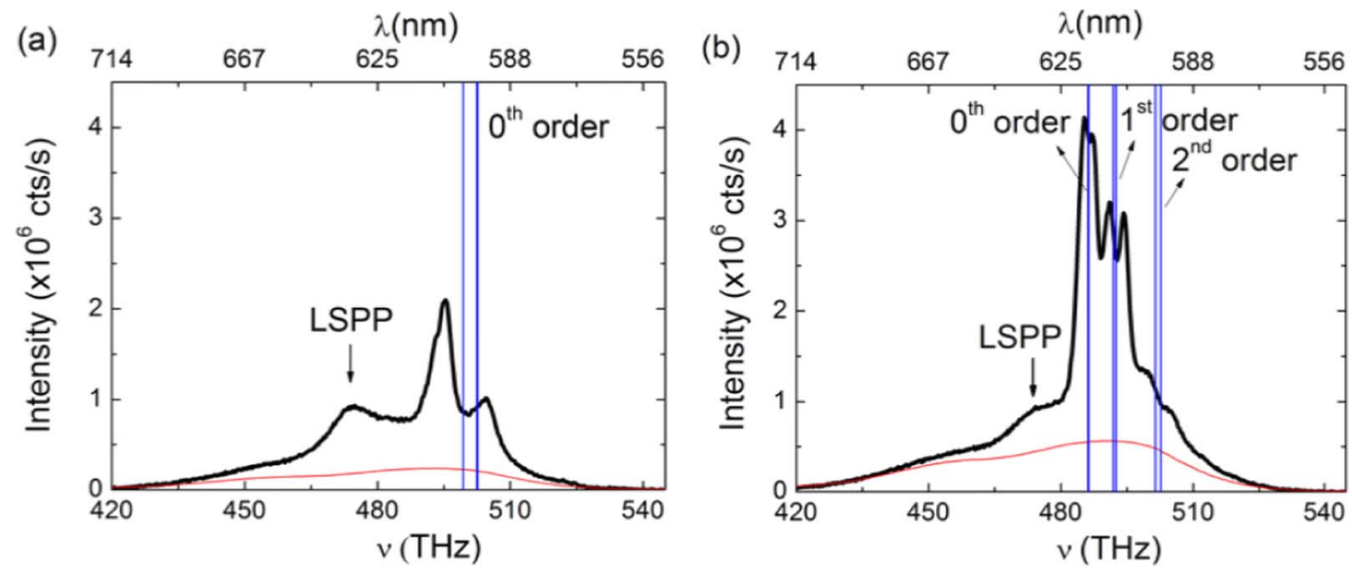

Figure 3. Photoluminescence spectra from the mixture of F305 and F083 dyes in a polymer layer deposited on the nanoparticle array with a period of 450 nm (black solid curve) and on top of the silica substrate (thin red curve) measured in the direction normal to the sample surface. (a) Corresponds to a layer thicknesses of $t=530 \mathrm{~nm}$, while (b) to a thickness of $t=1960 \mathrm{~nm}$. The vertical lines indicate the calculated frequencies of the TE and TM modes guided in polymer layers with these thicknesses. The orders of the modes are indicated in the figure. The LSPP frequency of the individual nanoparticles is indicated with the vertical arrow in both figures. 
(a)

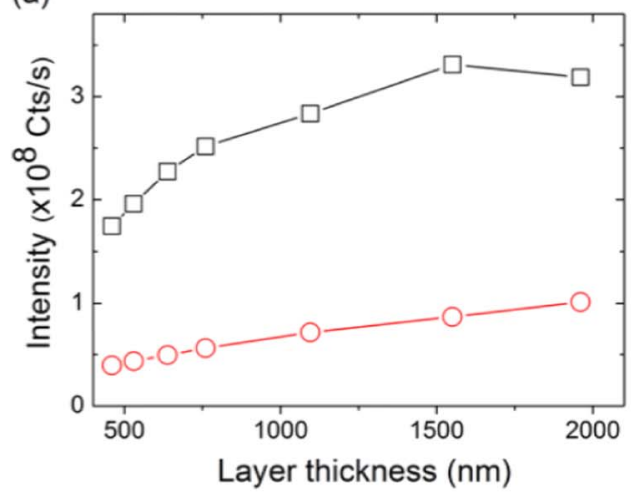

(b)

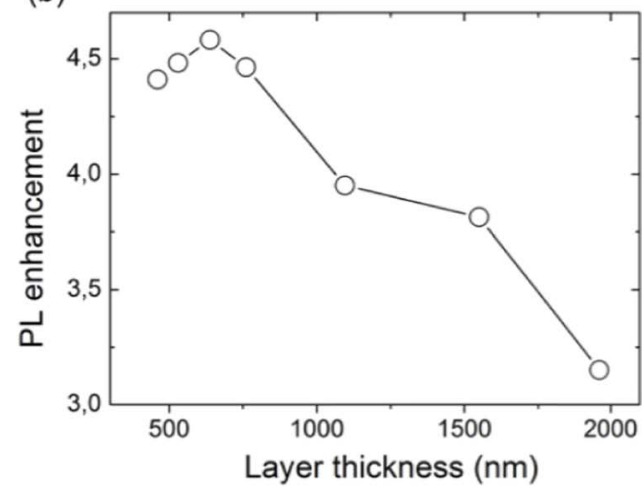

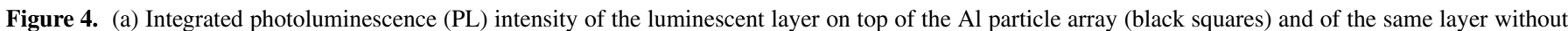

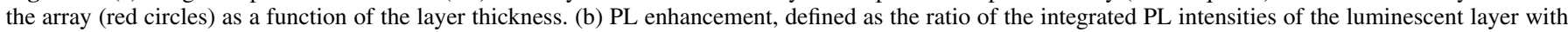
and without the particle array, as a function of the layer thickness. The solid lines are guides to the eye.

$\mathrm{TM}_{0}$ and $\mathrm{TE}_{0}$ quasi-guided modes are redshifted to $487 \mathrm{THz}$ and 485 $\mathrm{THz}$, respectively. This shift is consistent with the larger thickness and a better electromagnetic field confinement in the high refractive index medium, i.e., the waveguide. Besides the fundamental modes, higher order quasi-guided modes appear in the emission spectrum: The $\mathrm{TM}_{1}$ and $\mathrm{TE}_{1}$ at 494 and $491 \mathrm{THz}$, respectively, and the $\mathrm{TM}_{2}$ and $\mathrm{TE}_{2}$ modes at 505 and $499 \mathrm{THz}$. We also note that the discrepancy between the calculated eigenfrequencies and the measured peaks is smaller for the thicker waveguide. This is consistent with the previous explanation of this discrepancy in terms of the perturbation that the particles introduce to the guided modes. This perturbation should be reduced as the thickness of the waveguide increases. In addition to the narrow emission resonances assigned to the quasi-guided modes, we also observe in Figs. $3 \mathrm{a}$ and $3 \mathrm{~b}$ a broad peak at $474 \mathrm{THz}$. This peak does not significantly shift as the thickness of the luminescent layer is changed, which indicates that its origin is not in the waveguide. Instead, this broad peak can be assigned to the LSPPs in the individual $\mathrm{Al}$ particles of the array.

Figure 4a displays the integrated PL intensity of the reference layer (red circles) and of the luminescent metamaterial (black squares) as a function of the layer thickness. The integration is performed over the emission spectrum of the dye. For the reference layer we observe a monotonous increase of the emission due to the increased absorption of the blue light. For the luminescent metamaterial the dependence of the emission with the layer thickness is more complex. The integrated PL intensity is larger for all the thicknesses compared to the reference layer. This increased intensity is due to an enhanced blue absorption by the dye due to the scattering with the particle array and, as discussed next, to the coupling of the emission to the quasi-guided modes of the structure.

To better compare the PL of the luminescent metamaterials with the reference layers, we display in Fig. $4 \mathrm{~b}$ the integrated PL enhancement, defined as the ratio of the integrated PL intensity of the metamaterial to the reference, as a function of the layer thickness. In this figure we can appreciate the initial increase of PL enhancement for thin luminescent layers and the decrease of this quantity as the thickness is further increased. This behavior can be qualitatively explained by field overlap between the guided modes, the dye molecules and the particle array responsible for the outcoupling of the emission to free space. As discussed in Ref.,28 the field of the fundamental guided mode in thin layers is mainly distributed in the surrounding dielectric, i.e., at positions where the dye molecules and the particle array are not located. Therefore, the emission from the molecules cannot efficiently couple to the guided modes. As the layer thickness increases, the field is better confined in this layer and the fraction of the emission that can couple to the guided modes increases. This field also scatters from the particle array coupling to free space. This is the reason for the initial increase of the PL enhancement as the luminescent layer thickness is increased. After reaching a maximum PL enhancement for $\mathrm{t} \sim 650 \mathrm{~nm}$, this quantity decreases. The decrease can be attributed to the weaker interaction of the guided modes with the array of particles. For thick layers we expect that the field is mainly distributed inside the waveguide and the scattering with the particle array located at the edge of the waveguide is weak. In the limit of very thick layers, the PL enhancement should converge to one and the emission from the bare luminescent layer should not be significantly modified by the particle array.

Luminous efficacy of optical radiation. - After having established the dependence of the PL intensity with the thickness of the luminescent layer, we describe the spectral luminous efficacy, $\eta_{s}$, of the luminescent metamaterial. The physical properties of emitting materials are characterized by radiometric units (e.g. photon energy and optical power) at any frequency. The photometric quantities (e.g. $\eta_{s}$ ) are relevant when the emission is detected by the human eye. Therefore, the manipulation and optimization of these photometric quantities are of utmost importance for SSL.

The $\eta_{s}$ characterizes how well a light source produces visible light and it relates the spectral power of the source to the luminous flux, $\Phi_{\text {lum }}$, which is the measure of the perceived power of light by the human eye. The $\eta_{s}$ is given by ${ }^{29}$

$$
\eta_{s}=\Phi_{\text {lum }} / \int_{\nu 1}^{\nu 2} W(\nu) d \nu,
$$

where $W(v)$ is the power spectral density, which is proportional to the measured counts per unit time at frequency $\nu$, times the photon energy at this frequency. The integral in the former equation is performed over the emission spectrum of the dye. Therefore, this equation defines the red luminous efficacy. We stress that this efficacy refers to a spectral efficacy, i.e., it has units of lumens per visible watt instead of lumens per input optical watt or lumens per electrical watt. This quantity has no bearing on the absorption, down-conversion and emission efficiencies of the system.

The luminous flux is given by

$$
\Phi_{\text {lum }}=683 \int_{\nu 1}^{\nu 2} V(\nu) W(\nu) d \nu,
$$

where the factor of 683 , with units of lumens per watt, scales the result with the definition of lumen, and $V(v)$ is the photopic sensitivity curve of the eye. ${ }^{3}$

Figure 5 displays $\eta_{s}$ as a function of the layer thickness for the luminescent metamaterial and for the luminescent layer. The luminous efficacy is improved by using the nanoparticle array, reaching a 


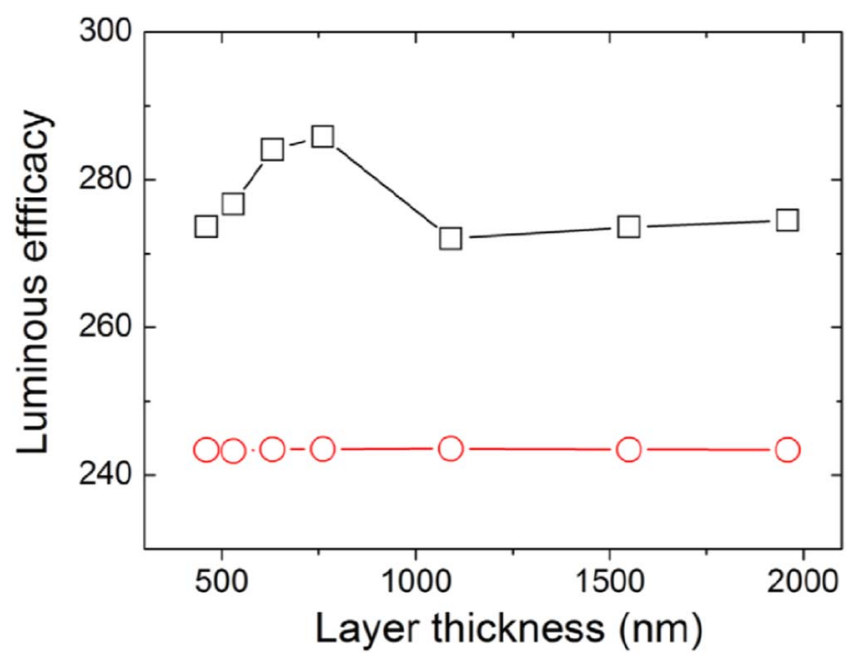

Figure 5. Luminous efficacy of the luminescent layer on top of the Al particle array (black squares) and of the same layer without the array (red circles) as a function of the layer thickness. The solid lines are guides to the eye.

maximum value of $286 \mathrm{~lm} / \mathrm{W}$ for $t=760 \mathrm{~nm}$ compared to the value of $245 \mathrm{~lm} / \mathrm{W}$ for the reference layer. Moreover, we see that the $\eta_{s}$ of the luminescent metamaterial shows a strong thickness dependence, in contrast to the $\eta_{s}$ of the bare luminescent layer, which is constant for all thicknesses.

We display in Fig. 6 the measured and normalized to the maximum emission spectrum, together with the photopic sensitivity curve, to understand better the behavior of $\eta_{s}$. Figure 6a corresponds to a bare luminescent layer with a thickness of $t=760 \mathrm{~nm}$. A relatively large area under $W(v)$ spectrally overlaps with the low frequency tail of $V(v)$, which limits the luminous efficacy. As pointed out in the previous section and shown in Figs. 6b-6d, the coupling of the emission to guided modes in the luminescent metamaterial strongly modifies the spectrum. This modification depends on the available modes in the layer to which the emission from the dye can couple, which is a function of the layer thickness. In this case we have that $W$ is not only a function of frequency but also of the layer thickness, i.e., $W(v, t)$. Since $V(v)$ is independent of the thickness, we can conclude that the $\eta_{s}$ becomes thickness dependent, as shown in Fig. 5. Indeed, the spectral position, linewidths, and intensities of the peaks in the spectrum can be varied by changing the thickness of the waveguide layer, which allows to control the overlap between $W(\nu, t)$ and $V(\nu)$. This is illustrated in Figs. 6b, 6c and 6d, which show the normalized emission spectra for $t=530 \mathrm{~nm}, t=760 \mathrm{~nm}$ and $t=1960 \mathrm{~nm}$, together with the photopic sensitivity curve (thin blue curves). The maximum value of $\eta_{s}$ is achieved for $t=760 \mathrm{~nm}$ (see Fig. 5). In this case the $\mathrm{TM}_{0}$ and $\mathrm{TE}_{0}$ guided modes are at $\nu=496$ and $491 \mathrm{THz}$, respectively, where $V=0.56$ and 0.5 . These modes provide a large contribution to the spectral power density in a narrow frequency range where $V(v)$ is relatively large. The decrease of $\eta_{s}$ for larger values of $t$ can be explained by the redshift of the $\mathrm{TM}_{0}$ and $\mathrm{TE}_{0}$ modes. For $t=1960 \mathrm{~nm}$ these modes are at $v=487 \mathrm{THz}$, where $V=0.43$, and at $v=485 \mathrm{THz}$, where $V=0.39$, i.e., at frequencies at which the values of $V$ are lower than for the $t=760 \mathrm{~nm}$ layer.

\section{Conclusions}

We have investigated the emission from luminescent layers on top of periodic arrays of metallic nanoparticles. The layers support guided modes to which the emission can couple. The arrays couple out the guided modes in directions that are defined by the lattice. The number of guided modes available for the emitter to couple can be controlled by changing the thickness of the layers. This change modifies the spectral content of the emission spectrum, which has an impact on
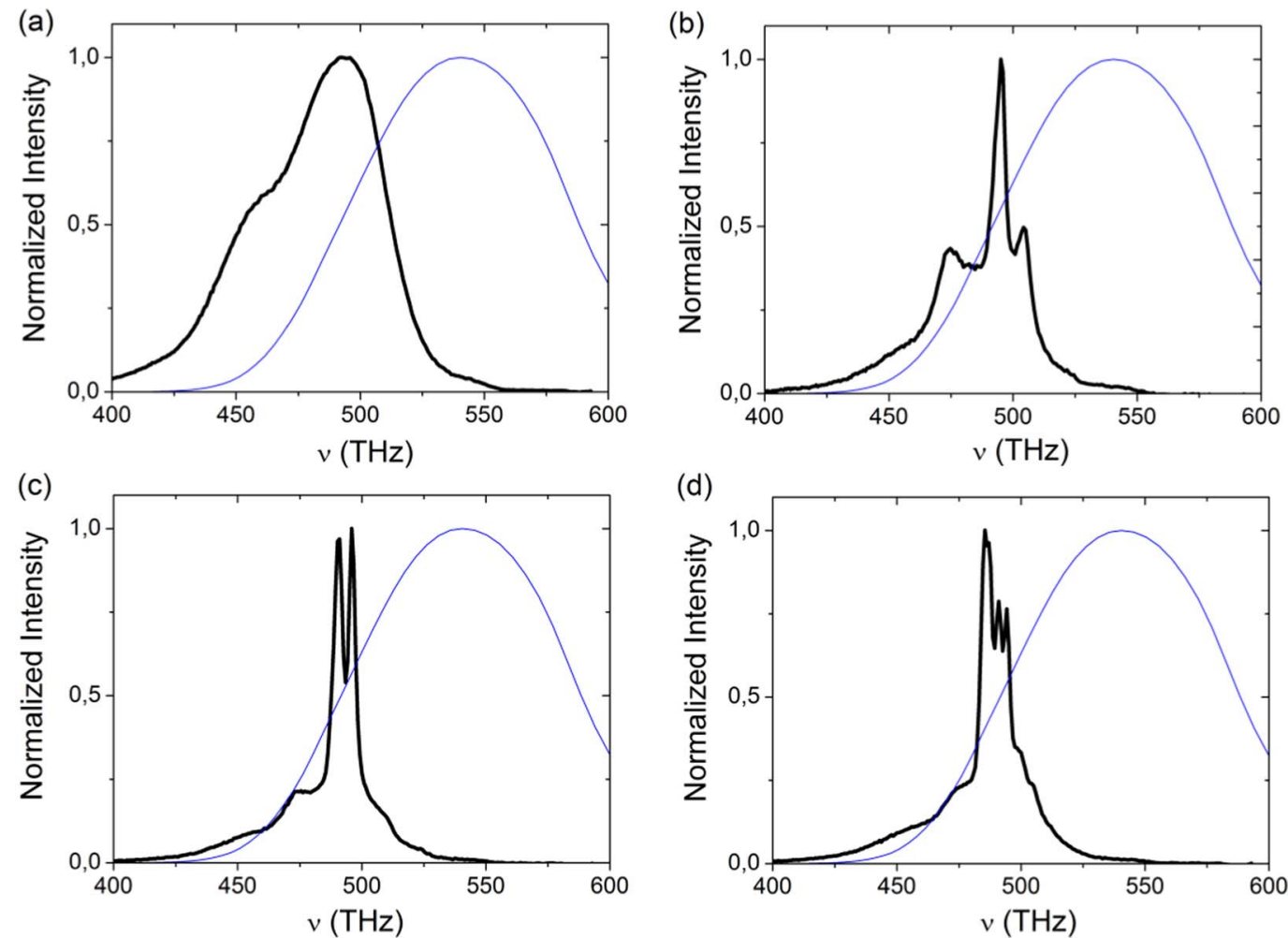

Figure 6. (a) Emission spectrum normalized to the maximum for a dye layer thickness of $760 \mathrm{~nm}$. (b), (c), and (d) display the normalized spectra of luminescent matematerials with layer thickness of $530 \mathrm{~nm}$ (b), $760 \mathrm{~nm}$ (c), and $1960 \mathrm{~nm}$ (d). The thin blue curves in all the panels represent the photopic sensitivity curve of the eye. 
the photoluminescence enhancement and on the luminous efficacy of the system. These type of luminescent layers coupled to particle arrays can be considered as luminescent metamaterials, with emission properties that are artificially designed by the structure rather than by the material. This additional degree of freedom can be employed to improve the emission characteristics of white LEDs.

\section{Acknowledgments}

We are thankful to M.A. Verschuuren, M. Lunz, D. de Boer, R. van Brakel for insightful discussions and for the preparation of the samples. This research was financially supported by the Nederlandse Organisatie voor Wetenschappelijk Onderzoek (NWO) through the project LEDMAP of the Technology Foundation STW and through the Industrial Partnership Program Nanophotonics for Solid State Lighting between Philips and the Foundation for Fundamental Research on Matter FOM.

\section{References}

1. S. Nakamura, S. Pearson, and G. Fasol, The Blue Laser Diode: The Complete History, 2nd Ed., Springer-Verlag Berlin-Heidelberg (2000).

2. E. F. Schubert, Light-Emitting Diodes, 2nd ed., Cambridge University Press, (2006).

3. J. J. Vos, Color Res. Appl., 3, 125 (1978).

4. H. Raether, Surface Plasmons on Smooth and Rough Surfaces and on Gratings, Springer Tracts in Modern Physics, Volume 111 (1988).

5. Palash Bharadwaj, Bradley Deutsch, and Lukas Novotny, Adv. Opt. Phot., 1, 438 (2009).

6. S. Murai, M. A. Verschuuren, G. Lozano, G. Pirruccio, S. R. K. Rodriguez, and J. Gómez Rivas, Opt. Express, 21, 4250 (2013).

7. A. Christ, S. G. Tikhodeev, N. A. Gippius, J. Kuhl, and H. Giessen, Phys. Rev. Lett., 91, 183901 (2003).
8. T. Zentgraf, S. Zhang, R. F. Oulton, and X. Zhang, Phys. Rev. B, 80, 195415 (2009).

9. K. T. Carron, W. Fluhr, M. Meier, A. Wokaun, and H. W. Lehmann, J. Opt. Soc. Am $B, 3,430$ (1986)

10. V. A. Markel, J. Opt. Soc. Am. B, 12, 1783 (1995).

11. S. Zou and G. C. Schatz, J. Chem. Phys., 121, 12606 (2004)

12. G. Vecchi, V. Giannini, and J. Gómez Rivas, Phys. Rev. Lett., 102, 146807 (2009).

13. V. Giannini, G. Vecchi, and J. Gómez Rivas, Phys. Rev. Lett., 105, 266801 (2010)

14. G. Pellegrini, G. Mattei, and P. Mazzoldi, J. Phys. Chem. C, 115, 24662 (2011).

15. S. R. K. Rodriguez, G. Lozano, M. A. Verschuuren, R. Gomes, K. Lambert B. de Geyter, A. Hassinen, D. van Thourhout, Z. Hens, and J. Gómez Rivas, Appl. Phys. Lett., 100, 111103 (2012)

16. S. R. K. Rodriguez, S. Murai, M. A. Verschuuren, and J. Gómez Rivas, Phys. Rev. Lett., 109, 166803 (2012).

17. T. V. Teperik and A. Degiron, Phys. Rev. Lett., 108, 147401 (2012).

18. W. Zhou, M. Dridi, J. Y. Suh, C. H. Kim, D. T. Co, M. R. Wasielewski, G. C. Schatz, and T. W. Odom, Nature Nanotechnol., 8, 506 (2013).

19. A. H. Schokker and A. F. Koenderink, Phys. Rev. B, 90, 155452 (2014)

20. G. Lozano, D. J. Louwers, S. R. K. Rodriguez, S. Murai, O. T. A. Jansen, M. A. Verschuuren, and J. Gómez Rivas, Light : Sci. Appl., 2, e66 (2013).

21. A. Abass, S. R. K. Rodriguez, T. Ako, T. Aubert, M. A. Verschuuren, D. van Thourhout, J. Beeckman, Z. Hens, J. Gómez Rivas, and B. Maes, Nano Lett., 14, 5555 (2014).

22. S. R. K. Rodriguez, F. Bernal Arango, T. P. Steinbusch, M. A. Verschuuren, A. F. Koenderink, and J. Gómez Rivas, Phys. Rev. Lett., 113, 247401 (2014).

23. M. A. Verschuuren, Substrate conformal imprint lithography for nanophotonics, $\mathrm{PhD}$ thesis, Utrecht University (2010).

24. Davy Gérard and Stephen K Gray, J. Phys. D: Appl. Phys., 48, 184001 (2015).

25. H. Kogelnik, in Integrated optics, T. Tamir, Editor, Springer-Verlag, Berlin (1979).

26. A. Yariv and P. Yeh, Photonics: Optical Electronics in Modern Communications, Oxford University Press, Chap. 3, New York (2006).

27. S. G. Tikhodeev, A. L. Yablonskii, E. A. Muljarov, N. A. Gippius, and T. Ishihara, Phys. Rev. B, 66, 045102 (2002).

28. S. R. K. Rodriguez, Y. T. Chen, T. P. Steinbusch, M. A. Verschuuren, A. F. Koenderink, and J. Gómez Rivas, Phys. Rev. B, 90, 235406 (2014).

29. T. W. Murphy, J. Appl. Phys., 111, 104909 (2012). 